https://doi.org/10.23913/ride.v11i21.798

Artículos científicos

\title{
Conocimientos, actitudes y prácticas socioambientales en estudiantes de la Universidad Autónoma de Guerrero, México
}

\author{
Knowledge, attitudes and socio-environmental practices in students of the \\ Autonomous University of Guerrero, Mexico
}

\section{Conhecimentos, atitudes e práticas socioambientais em alunos da Universidade Autônoma de Guerrero, México}

\author{
Herlinda Gervacio Jiménez \\ Universidad Autónoma de Guerrero, México \\ lindagj09@gmail.com \\ https://orcid.org/0000-0003-3037-9528 \\ Benjamín Castillo Elías* \\ Universidad Autónoma de Guerrero, México \\ bcastilloe@hotmail.com \\ https://orcid.org/0000-0003-1487-5353 \\ * Autor de correspondencia
}

\section{Resumen}

Esta investigación analizó el nivel de educación ambiental que poseen estudiantes del nivel medio superior de la Universidad Autónoma de Guerrero, se consideraron las variables sobre el conocimiento y formación ambiental, saber actuar ante los problemas socioambientales, prácticas de carácter ambiental, percepción sobre la complejidad de problemas socioambientales, grado de ambientalización en el currículo, actitudes y conductas proambientales desarrolladas durante el proceso de enseñanza-aprendizaje. El objetivo de esta investigación fue explorar el conocimiento, la formación, las actitudes y conductas proambientales que tienen estudiantes de preparatoria, con base en la enseñanza-aprendizaje adquirido durante su estancia en el centro educativo de la Universidad Autónoma de Guerrero. El método utilizado fue eminentemente cuantitativo, y las 
variables fueron analizadas a través de la escala de Likert el cual es un método de investigación de campo que permite medir la opinión sobre un tema a través de una encuesta que identifica el grado de acuerdo o desacuerdo de cada pregunta, empleando 5 categorías para esta investigación. Los resultados indicaron que sus conocimientos y prácticas sobre temáticas ambientales van de "escasos" a "regular"; habitualmente saben actuar ante las problemáticas socioambientales que los rodea; se observó que poseen escasa percepción sobre la complejidad de los actuales problemas ambientales, el estudio demostró, que los estudiantes tienen escaza conciencia para actuar, cuidar y proteger su entorno socioambiental, con un bajo interés para resolver las problemáticas ambientales a nivel local, lo que se refleja en los resultados de la variable ambientalización en el currículo, que va de "escaso" a "regular". Se concluyó que la percepción de los estudiantes no impacta en acciones positivas a favor del ambiente y que estas acciones beneficien a los centros educativos y a su comunidad. Los datos de este estudio coinciden con otras investigaciones similares a ésta, donde se ha llegado a la misma conclusión, el conocimiento y la conciencia no han sido suficientes para desarrollar actitudes proambientales, es necesario poner y llevar a la práctica acciones responsables, con una adecuada metodología para proponer actividades que ayuden a la resolución de los problemas ambientales vigentes y que estas acciones se vean reflejadas en beneficio del entorno en el que los estudiantes se desenvuelven.

Palabras clave: ambientalización, centros educativos, educación ambiental, currículo, transverzalización.

\section{Abstract}

This research analyzed the level of environmental education that students have in the high school of the Autonomous University of Guerrero, were considered variables on environmental knowledge and training, how to act on socio-environmental problems, environmental practices, perception of the complexity of socio-environmental problems, degree of environmentalisation in the curriculum, attitudes and pro-environmental behaviors developed during the teaching-learning process. The objective of this research was to explore the knowledge, training, attitudes, and proenvironmental behaviors that high school students have, based on the teaching-learning acquired during their stay at the educational center of the Autonomous University of Guerrero. The method used was eminently quantitative, and the variables were analyzed through the Likert scale, which is a field research method that allows measuring the opinion on a topic through a survey that identifies the degree of agreement or disagreement of each question, using 5 categories for this 


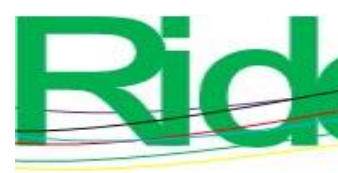

Revista Iberoamericana para la Investigación y el Desarrollo Educativo ISSN $2007-7467$

research. The results indicated that their knowledge and practices on environmental issues range from "scarce" to "regular"; they usually know how to deal with the socio-environmental problems that surround them; it was observed that they have a little perception about the complexity of current environmental problems, the study showed that students have limited awareness to act, care and protect their socio-environmental environment, with a low interest in solving environmental problems at the local level, which is reflected in the results of the environmentalization variable in the curriculum, which ranges from "scarce" to "regular". It was concluded that the students' perception does not impact on positive actions in favor of the environment and that these actions benefit the educational centers and their community. The data from this study coincide with other research similar to this one, where the same conclusion has been reached, knowledge and awareness have not been enough to develop pro-environmental attitudes, it is necessary to put and implement responsible actions, with an adequate methodology to propose activities that help to solve current environmental problems and that these actions are reflected in the benefit of the environment in which the students work.

Keywords: environmentalization, educational centers, environmental education, curriculum, transverzalization.

\section{Resumo}

Esta pesquisa analisou o nível de educação ambiental dos alunos do ensino médio da Universidade Autônoma de Guerrero, foram consideradas as variáveis sobre o conhecimento e a formação ambiental, saber agir perante os problemas socioambientais, práticas de caráter ambiental, percepção sobre a complexidade dos problemas socioambientais, grau de ambientalização no currículo, Atitudes e condutas pró-ambientais desenvolvidas durante o processo de ensinoaprendizagem. O objectivo desta investigação era explorar os conhecimentos, formação, atitudes e comportamentos pró-ambientais dos estudantes do ensino secundário, com base no ensinoaprendizagem adquirido durante a sua estadia no centro educativo da Universidade Autónoma de Guerrero. O método utilizado foi eminentemente quantitativo, e as variáveis foram analisadas através da escala Likert que é um método de pesquisa de campo que permite medir a opinião sobre um tópico através de um inquérito que identifica o grau de concordância ou desacordo de cada questão, utilizando 5 categorias para esta pesquisa. Os resultados indicaram que os seus conhecimentos e práticas sobre questões ambientais variam de "escassos" a "regulares"; normalmente sabem como lidar com os problemas socioambientais que os rodeiam; observou-se 
que têm pouca percepção da complexidade dos problemas ambientais actuáis, o estudo mostrou que os estudantes têm pouca consciência para agir, cuidar e proteger o seu ambiente sócioambiental, com um baixo interesse em resolver problemas ambientais a nível local, o que se reflecte nos resultados da variável de ambientalização no currículo, que varia de "baixa" a "regular". Concluiu-se que a percepção dos estudantes não tem impacto nas acções positivas a favor do ambiente e que estas acções beneficiam os centros educativos e a sua comunidade. Os dados deste estudo são coerentes com outras investigações semelhantes a este, onde se chegou à mesma conclusão, o conhecimento e a sensibilização não foram suficientes para desenvolver atitudes próambientais, é necessário pôr em prática acções responsáveis, com uma metodologia adequada para propor actividades que ajudem a resolver os problemas ambientais actuais e que estas acções se reflictam no benefício do ambiente em que os estudantes trabalham.

Palavras-chave: ambientalização; centros educativos; educação ambiental; curriculum; transverzalização.

\section{Introducción}

En diversas conferencias mundiales organizadas por las Naciones Unidas para tratar temas como la educación para el desarrollo sustentable, la educación ambiental o la educación para la ciudadanía mundial, ha habido consenso sobre la importancia de esos temas para formar personas dotadas de actitudes y conocimiento necesarios para afrontar desafíos relacionados con problemas socioambientales (Organización de las Naciones Unidas para la Educación, la Ciencia y la Cultura [Unesco], 2014, 2015). De hecho, se han establecido diferentes políticas y agendas ambientales donde se dota de estrategias y recomendaciones a los líderes mundiales sobre la importancia de contar con una agenda ambiental basada en las necesidades y problemáticas de cada país.

En el plano educativo, las universidades de México no han estado ajenas a estos lineamientos, de tal manera que se han adherido a atender las recomendaciones de la Unesco para contribuir a la reorientación de procesos sustantivos y del fortalecimiento de las capacidades institucionales en el desarrollo de tecnología y la aplicación de conocimiento con el firme compromiso de mantener en el tiempo estos compromisos (Organización Universitaria Interamericana-Organización Interamericana para la Educación Superior [OUI-IOHE], 2011).

La Secretaría de Educación Pública (SEP), dentro de los fundamentos legales de la actual reforma educativa en el nivel medio superior (NMS) en México, ha establecido como objetivo 


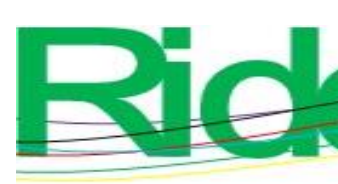

Revista Iberoamericana para la Investigación y el Desarrollo Educativo ISSN $2007-7467$

primordial asegurar que el estudiante reciba independientemente de su entorno socioeconómico, origen étnico o género, una educación que proporcione aprendizajes y conocimientos significativos, relevantes y útiles para la vida. La intención es formar ciudadanos que tengan la motivación y capacidad de lograr su desarrollo personal, laboral y familiar, dispuestos a mejorar su entorno social y natural (Secretaría de Educación Pública [SEP], 2017). Por ello, se define un nuevo perfil de egreso conformado por once ámbitos que establecen las competencias que deben caracterizar a todos los egresados de la EMS, como el cuidado del medio ambiente, para lo cual se debe "comprender la importancia de la sustentabilidad y asumir una actitud proactiva para encontrar soluciones sustentables. Pensar globalmente y actuar localmente. Valorar el impacto social y ambiental de las innovaciones e impactos científicos” (Secretaría de Educación Pública [SEP], 2017).

En tal sentido, debe quedar claro que la educación ambiental aborda como objeto de estudio las relaciones sociales con el medio ambiente, delimitando aquellos elementos que lo forman y construyendo sus objetivos. Esa delimitación da lugar a las acciones educativas, mediadas por las esferas de las relaciones personales, sociales y con el ambiente biofísico, que son recorridas por los ejes de impacto ambiental no favorable "negativo" y favorable "positivo" (Calixto, 2018).

En efecto, las problemáticas ambientales existentes a nivel mundial, las cuales se han recrudecido en todos los ámbitos sociales y económicos, y están afectando al planeta con la desertificación, la escasez de agua, la sobreexplotación de los recursos naturales, el consumismo excesivo, entre otros, ha originado la búsqueda de respuestas desde la docencia. Calixto (2019), propone estrategias didácticas en las instituciones educativas para la mitigación de las problemáticas ambientales existentes.

La educación ambiental no solo comprende los conocimientos sobre los problemas ambientales, sino también la sensibilización, la adquisición de conciencia, las aptitudes y comportamientos que están estrechamente relacionados con los valores y las actitudes del ser humano. Las universidades como formadoras y modeladoras de conductas y actitudes tienen la responsabilidad de garantizar aprendizajes significativos en los estudiantes sobre el tema que aquí se aborda.

Por ello, en la presente investigación se explora el nivel de conocimientos, actitudes y conductas que poseen los estudiantes en favor del cuidado del ambiente. La presente investigación se ha sustentado en distintos estudios efectuados en diferentes momentos y tiempos en los Campus 


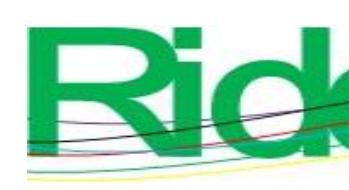
Revista Iberoamericana para la
Investigación y el Desarrollo Educativo
ISSN $2007-7467$

universitarios, y cuyos resultados han servido como base para fomentar cambios pertinentes en el currículo de las universidades de México y de todo el mundo.

En México, los programas académicos vinculados con la educación ambiental, las políticas para la sustentabilidad de las instituciones de educación superior y la importancia de la formación de educadores ambientales en México, se iniciaron hace aproximadamente veinte años (GonzálezGaudiano y Arias-Ortega, 2017; Martínez-Fernández y González, 2015). Al respecto, Benayas del Álamo, Alba y Sánchez (2002) infieren que la ambientalización de los Campus universitarios requiere de recursos necesarios para su correcta implementación, que transfieran un proceso de cambio en los universitarios y que funcione como un efecto replicador en la sociedad.

Investigaciones como las realizadas por Leff (2006), Tovar-Gálvez (2012), Medina y Páramo (2014), Peza (2014), Gervacio y Castillo (2019), han contribuido en los procesos educativos en el ámbito socioambiental; de igual manera, en la región latinoamericana se han venido desarrollando diversas investigaciones y estudios sobre el tema y su integración en las universidades como una tendencia en la contribución con la sustentabilidad ambiental (Carrasco y Vásquez, 2016; Zúñiga-Sánchez y Marúm-Espinosa, 2016).

De tal suerte que, el objetivo de la presente investigación ha sido la exploración del conocimiento, la formación, las actitudes y las conductas proambientales de los estudiantes de preparatoria, basados en la enseñanza-aprendizaje y los conocimientos adquiridos sobre temáticas socioambientales, sus posibles soluciones o propuestas que permitan mantener un equilibrio entre los impactos antropogénicos y la armonía con el ambiente que los rodea.

\section{Metodología}

Los procesos de diseño metodológico dependen de la perspectiva epistemológica con que se aborde el diagnóstico que se quiere realizar, así como de los propósitos establecidos (Yazan, 2015). En este sentido, la metodología que se utilizó para la presente investigación fue a través de encuestas aplicadas a estudiantes de cuatro preparatorias de la Universidad Autónoma de Guerrero (UAGro), ubicadas en Acapulco, Guerrero (México).

\section{Área de estudio}

Se trabajó con las preparatorias 2, 7, 17 y 27 del nivel medio superior de la Universidad Autónoma de Guerrero ubicadas en la ciudad de Acapulco, Guerrero (figura 1). 


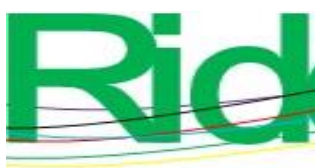

Revista Iberoamericana para la Investigación y el Desarrollo Educativo ISSN 2007 - 7467

Figura 1. Mapa de ubicación de las preparatorias encuestadas de la UAGro

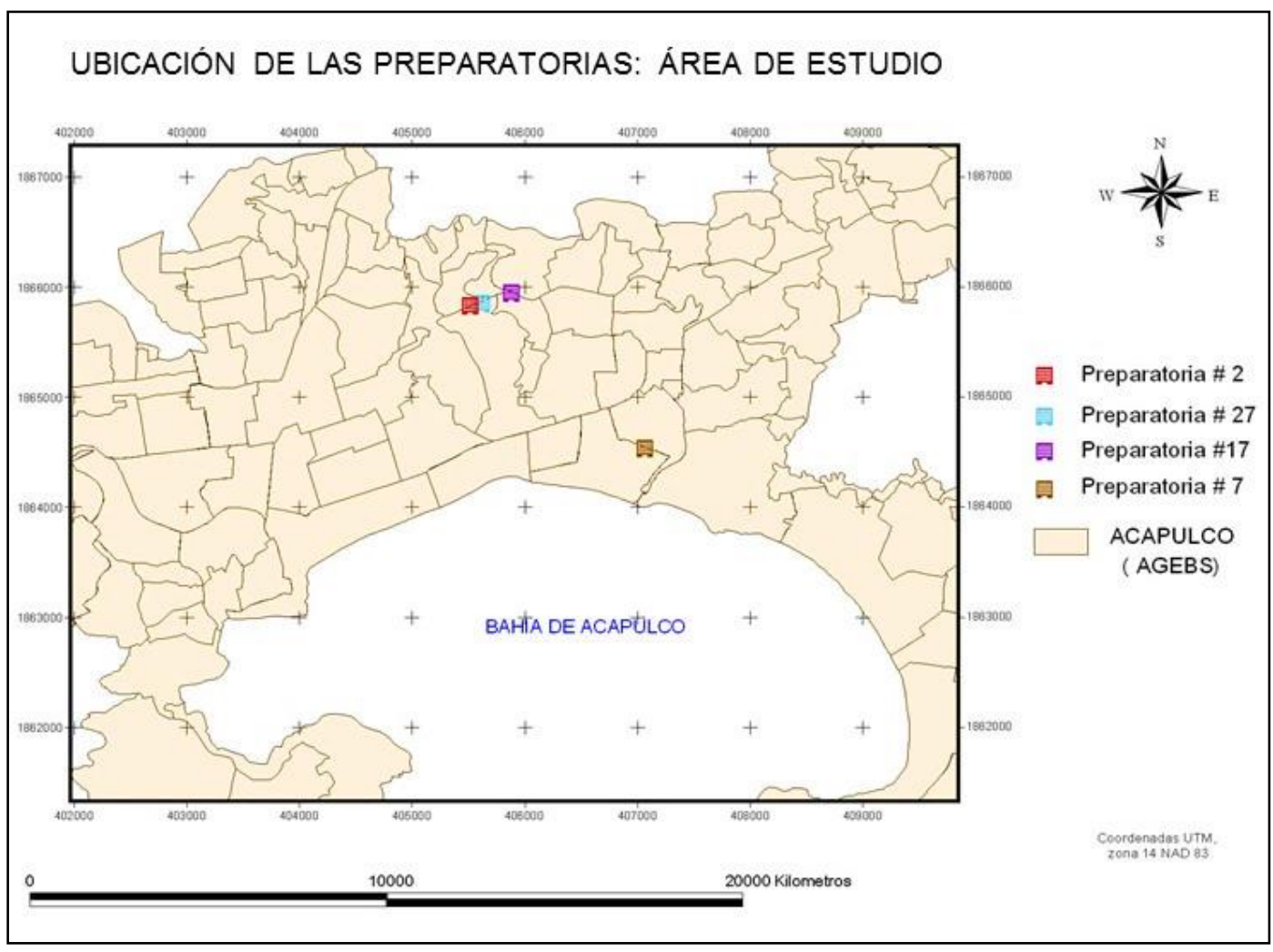

Fuente: Elaboración propia

\section{Parte experimental}

Las encuestas se aplicaron en los salones de clase a 1,104 estudiantes del sexto semestre distribuidos en las cuatro preparatorias en los turnos matutino y vespertino; se consideró encuestar solo a estudiantes del tercer año porque ya habían tomado prácticamente todas las materias del plan de estudios; esto permitió identificar los conocimientos y la formación ambiental que adquirieron durante su trayecto por la preparatoria.

La encuesta fue considerada el método más apropiado porque las dos variables tenían que ver con "el grado de...", lo cual es una cuestión de carácter eminentemente cuantitativo. Dichas variables, a su vez, son de carácter ordinal. En otras palabras, no fueron las predisposiciones del investigador las que determinaron el diseño de la investigación (Weigel y Weigel, 1978; Wences, 2005).

Para analizar los datos obtenidos se aplicaron sobre las variables las posibilidades que ofrecía la estadística descriptiva. El vaciado de datos y la construcción de escalas de la encuesta se realizó en el paquete estadístico SPSS (Statistical Package for Social Sciences, v. 15) (Pérez, 2009). 


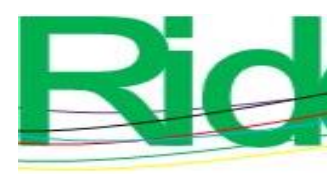

Revista Iberoamericana para la
Investigación y el Desarrollo Educativo
ISSN $2007-7467$

Las variables se analizaron a través de escalas Likert (Hernández-Sampieri, Fernández y Baptista, 2014). Este software se aplicó para la depuración de ítems, así como la formación de escalas, de acuerdo con el método alfa de Cronbach, se consideraron las siguientes variables:

- Conocimientos de formación ambiental recibidos en la escuela.

- Saber actuar ante problemas socioambientales que se presentan en la vida.

- Prácticas de carácter ambiental realizadas con los profesores.

- Percepción sobre la complejidad de los problemas socioambientales.

- Ambientalización en el currículo.

Para analizar la variable ambientalización del currículo, la encuesta aplicada a los estudiantes contempló un bloque referente al grado de ambientalización del plan de estudios, para ello, se incluyeron todas las asignaturas cursadas hasta el sexto semestre. Para el análisis se emplearon cinco escalas que iban de "excelente" a "nulo".

\section{Resultados}

El primer análisis corresponde a la variable preparatorias como variable independiente, y la variable formación ambiental recibida en la escuela como dependiente. En este caso interesa saber qué piensan los estudiantes de cada una de las preparatorias en relación con el conocimiento y formación ambiental obtenida en las escuelas.

En este sentido, se observó que el mayor porcentaje de las respuestas de los estudiantes se ubicaba en la categoría "regular". El análisis mostró que estadísticamente no existe asociación entre ambas variables $($ Chi-cuadrado $=11.427 ; \mathrm{P}=0.493)$, con una relación muy débil $(\mathrm{d}$ de Somers $=$ $0.030, \mathrm{P}=0.242$ ), por lo que independientemente de la preparatoria de que se trate, el conocimiento y la formación ambiental es regular con una tendencia a ser buena (tabla 1). 


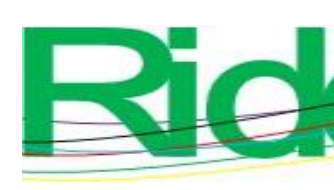

Revista Iberoamericana para la Investigación y el Desarrollo Educativo ISSN $2007-7467$

Tabla 2. Opinión de género con relación al conocimiento y formación ambiental recibida

\begin{tabular}{|c|c|c|c|c|c|c|c|c|c|c|c|c|c|}
\hline & & \multicolumn{10}{|c|}{$\begin{array}{l}\text { Conocimiento y formación ambiental recibida en la } \\
\qquad \text { escuela }^{2}\end{array}$} & \multicolumn{2}{|c|}{ Total } \\
\hline & & \multicolumn{2}{|c|}{ Nula } & \multicolumn{2}{|c|}{ Escasa } & \multicolumn{2}{|c|}{ Regular } & \multicolumn{2}{|c|}{ Buena } & \multicolumn{2}{|c|}{ Excelente } & \multirow{2}{*}{$\%$} & \multirow{2}{*}{$\mathrm{n}$} \\
\hline & & $\%$ & $\mathrm{n}$ & $\%$ & $\mathrm{n}$ & $\%$ & $\mathrm{n}$ & $\%$ & $\mathrm{n}$ & $\%$ & $\mathrm{n}$ & & \\
\hline \multirow{2}{*}{ Género $^{I}$} & Hombre & 2 & 10 & 20 & 97 & 52 & 252 & 24 & 114 & 2 & 10 & 100 & 483 \\
\hline & Mujer & 2 & 14 & 23 & 140 & 50 & 311 & 25 & 152 & 0 & 4 & 100 & 621 \\
\hline
\end{tabular}

Chi-cuadrado de Pearson $(\mathrm{x} 2)=5.487, \mathrm{gl}=4$, Sig $=.241, \mathrm{~d}=.024, \mathrm{Sig}=.394$

${ }^{1}$ Variable Independiente $\&^{2}$ Variable dependiente

Fuente: Elaboración propia

El análisis de preparatorias como variable independiente, y saber actuar ante problemas socioambientales que se presentan en la vida diaria como variable dependiente se realizó con la finalidad de conocer en qué medida actúan los estudiantes de las distintas preparatorias ante los problemas ambientales, y cuál preparatoria destaca más en este sentido.

Los resultados demostraron que "frecuentemente" saben actuar ante los problemas socioambientales que se presentan en la vida diaria, aunque las pruebas estadísticas manifestaron que no existe asociación entre dichas variables (Chi-cuadrado $=9.472 ; \mathrm{P}=0.662$ ) con una relación sumamente débil ( $\mathrm{d}$ de Somers $=0.050, \mathrm{P}=0.44$ ); por lo tanto, independientemente de la preparatoria de que se trate, los estudiantes habitualmente saben actuar ante los problemas socioambientales que se presentan en su vida diaria (tabla 3 ). 


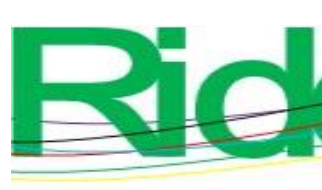

Revista Iberoamericana para la Investigación y el Desarrollo Educativo ISSN 2007 - 7467

Tabla 3. Preparatorias de la UAGro con relación a saber actuar ante problemas socioambientales

\begin{tabular}{|c|c|c|c|c|c|c|c|}
\hline & \multicolumn{5}{|c|}{$\begin{array}{c}\text { Saber actuar ante problemas socioambientales que se } \\
\text { presentan en la vida diaria }\end{array}$} & \multirow[b]{2}{*}{$\begin{array}{c}\text { Total } \\
\%\end{array}$} \\
\hline & & $\begin{array}{c}\text { Nunca } \\
\%\end{array}$ & $\begin{array}{c}\text { Raramente } \\
\%\end{array}$ & $\begin{array}{c}\text { Algunas } \\
\text { veces } \\
\%\end{array}$ & $\begin{array}{l}\text { Frecuentemente } \\
\%\end{array}$ & $\underset{\%}{\text { Siempre }}$ & \\
\hline \multirow{4}{*}{$\begin{array}{l}\text { Preparatorias } \\
\text { de la UAGro } \\
\text { del municipio } \\
\text { de Acapulco, } \\
\text { Guerrero. }{ }^{1}\end{array}$} & $\begin{array}{l}\text { Preparatoria } \\
\text { nro. } 2\end{array}$ & 2 & 10 & 30 & 41 & 17 & 100 \\
\hline & $\begin{array}{l}\text { Preparatoria } \\
\text { nro. } 7\end{array}$ & 2 & 11 & 33 & 36 & 18 & 100 \\
\hline & $\begin{array}{l}\text { Preparatoria } \\
\text { nro.17 }\end{array}$ & 3 & 12 & 33 & 38 & 14 & 100 \\
\hline & $\begin{array}{l}\text { Preparatoria } \\
\text { nro. } 27\end{array}$ & 4 & 10 & 35 & 39 & 12 & 100 \\
\hline
\end{tabular}

Chi-cuadrado de Pearson $(x 2)=9.472, \mathrm{gl}=12, \mathrm{Sig}=.662, \mathrm{~d}=.050, \mathrm{Sig}=.044$

1 Variable Independiente \& 2 Variable dependiente

Fuente: Elaboración propia

El análisis de la variable género en relación con la variable saber actuar ante los problemas socioambientales que se presentan en la vida diaria, se demostró que los hombres manifestaron que de acuerdo con su formación y conocimiento su actuación es de "buena" a "regular", mientras que las mujeres consideraron tener una "buena" actuación ante los problemas socioambientales. Las pruebas estadísticas revelaron que no existe asociación entre las variables (Chi-cuadrado $=7.866 ; \mathrm{P}=0.097)$ con una relación débil $(\mathrm{d}$ de Somers $=0.044, \mathrm{P}=0.102)$, por lo que al igual que en otros casos, ambos géneros de estudiantes, consideraron tener una "buena" actuación ante los problemas socioambientales que se presentan en su vida diaria (tabla 4).

Tabla 4. Opinión de género con relación al saber actuar ante problemas socioambientales

\begin{tabular}{|c|c|c|c|c|c|c|c|c|c|c|c|c|c|}
\hline & \multicolumn{10}{|c|}{$\begin{array}{c}\text { Saber actuar ante problemas socioambientales que se } \\
\text { presentan en la vida diaria }\end{array}$} & \multicolumn{2}{|c|}{ Total } \\
\hline & & \multicolumn{2}{|c|}{ Nula } & \multicolumn{2}{|c|}{ Escasa } & \multicolumn{2}{|c|}{ Regular } & \multicolumn{2}{|c|}{ Buena } & \multicolumn{2}{|c|}{ Excelente } & \multirow[b]{2}{*}{$\%$} & \multirow[b]{2}{*}{$\mathrm{n}$} \\
\hline & & $\%$ & $\mathrm{n}$ & $\%$ & $\mathrm{~N}$ & $\%$ & $\mathrm{~N}$ & $\%$ & $\mathrm{n}$ & $\%$ & $\mathrm{n}$ & & \\
\hline \multirow{2}{*}{ Género $^{I}$} & Hombre & 4 & 18 & 10 & 46 & 36 & 172 & 38 & 181 & 14 & 66 & 100 & 483 \\
\hline & Mujer & 2 & 12 & 11 & 70 & 31 & 191 & 39 & 244 & 17 & 104 & 100 & 621 \\
\hline
\end{tabular}

Chi-cuadrado de Pearson $(x 2)=7.866, \mathrm{gl}=4, \mathrm{Sig}=.097, \mathrm{~d}=.044, \mathrm{Sig}=.102$

${ }^{1}$ Variable Independiente $\&^{2}$ Variable dependiente.

Fuente: Elaboración propia 


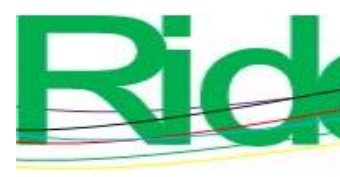

Revista Iberoamericana para la Investigación y el Desarrollo Educativo ISSN 2007 - 7467

El análisis de preparatorias como variable independiente y prácticas de carácter ambiental como variable dependiente se realizó con la finalidad de conocer en qué medida se llevan a cabo prácticas de carácter ambiental que amplíen el conocimiento y fortalezcan la formación de los estudiantes de las distintas preparatorias.

Los estudiantes contestaron que "algunas veces" llegan a realizar prácticas. Las pruebas estadísticas manifestaron que existe asociación entre las variables (Chi-cuadrado = 22.107; $\mathrm{P}=0.036)$ con una fuerte relación ( $\mathrm{d}$ de Somers $=0.062, \mathrm{P}=0.014)$, por lo que aquellos estudiantes que estudian en las preparatorias 2 y 7 consideraron que las prácticas se realizan "algunas veces" o "raramente", mientras que los estudiantes de las preparatorias 17 y 27 consideraron que "algunas veces" o "frecuentemente" llegan a desarrollar prácticas de carácter ambiental (tabla 5).

Tabla 5. Preparatorias de la UAGro con relación a las prácticas de carácter ambiental

\begin{tabular}{|l|c|c|c|c|c|c|}
\hline & \multicolumn{5}{|c|}{ Prácticas de carácter ambiental $^{2}$} & \\
\hline $\begin{array}{l}\text { Preparatorias de la } \\
\text { UAGro del municipio } \\
\text { de Acapulco, } \\
\text { Guerrero. }\end{array}$ & $\begin{array}{c}\text { Nunca } \\
\%\end{array}$ & $\begin{array}{c}\text { Raramente } \\
\%\end{array}$ & $\begin{array}{c}\text { Algunas } \\
\text { veces \% }\end{array}$ & $\begin{array}{c}\text { Frecuentemente } \\
\%\end{array}$ & $\begin{array}{c}\text { Siempre } \\
\%\end{array}$ & $\begin{array}{c}\text { Total } \\
\%\end{array}$ \\
\hline Preparatoria nro. 2 & 8 & 35 & $\mathbf{3 6}$ & 17 & 4 & 100 \\
\hline Preparatoria nro. 7 & 9 & 33 & $\mathbf{3 8}$ & 17 & 4 & 100 \\
\hline Preparatoria nro. 17 & 7 & 24 & $\mathbf{3 7}$ & 26 & 7 & 100 \\
\hline Preparatoria nro. 27 & 10 & 28 & $\mathbf{3 8}$ & 21 & 4 & 100 \\
\hline
\end{tabular}

Chi-cuadrado de Pearson $\left(\mathrm{x}^{2}\right)=22.107, \mathrm{gl}=12, \mathrm{Sig}=.036, \mathrm{~d}=.062, \mathrm{Sig}=.014$

${ }^{I}$ Variable Independiente $\&^{2}$ Variable dependiente.

Fuente: Elaboración propia

El análisis de preparatorias como variable independiente con relación a la percepción sobre la complejidad de los problemas socioambientales como variable dependiente se realizó con la finalidad de conocer en qué medida los estudiantes de cada una de las preparatorias tiene una percepción idónea sobre la complejidad actual de la problemática socioambiental.

Con un porcentaje por debajo de la media, los estudiantes encuestados indicaron ser perceptibles sobre la complejidad de los problemas ambientales. Las pruebas estadísticas revelaron que no existe asociación entre las variables de estudio (Chi-cuadrado $=7.884 ; \mathrm{P}=0.794$ ) con una relación muy débil $(\mathrm{d}$ de Somers $=0.007, \mathrm{P}=0.792$ ), por lo que, independientemente de la preparatoria donde estudien, la percepción sobre la complejidad de los problemas socioambientales va sobre "algunas veces" a "frecuentemente" (tabla 6). 


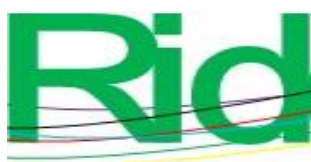

Revista Iberoamericana para la Investigación y el Desarrollo Educativo ISSN 2007 - 7467

Tabla 6. Preparatorias de la UAGro con relación a la percepción de problemas socioambientales

\begin{tabular}{|l|c|c|c|c|c|c|}
\hline & \multicolumn{5}{|c|}{ Percepción sobre la complejidad de los problemas } & \\
socioambientales
\end{tabular}

Chi-cuadrado de Pearson $(x 2)=7.884, \mathrm{gl}=12, \mathrm{Sig}=.794, \mathrm{~d}=.007, \mathrm{Sig}=.792$

${ }^{1}$ Variable Independiente $\&^{2}$ Variable dependiente.

Fuente: Elaboración propia

El análisis de la variable género en relación con la variable percepción sobre la complejidad actual de los problemas socioambientales se realizó con la finalidad de conocer en qué medida los hombres y mujeres de las escuelas conocen o están informados acerca de la problemática socioambiental actual. Tanto hombres como mujeres manifestaron que tienen suficiente conocimiento sobre la complejidad de los problemas socioambientales.

Las pruebas estadísticas determinaron que no existe asociación significativa entre las variables (Chi-cuadrado = 4.79; $\mathrm{P}=0.290)$ con una relación débil ( $\mathrm{d}$ de Somers = 0.026, $\mathrm{P}=0.335)$, por lo que independientemente del género, los estudiantes "frecuentemente" perciben la problemática socioambiental que viven en el momento (tabla 7). 


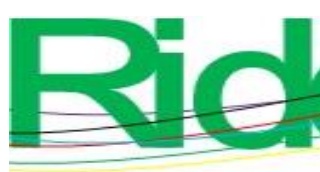

2

Revista Iberoamericana para la Investigación y el Desarrollo Educativo ISSN 2007 - 7467

Tabla 7. Opinión de género con relación a la percepción de los problemas socioambientales

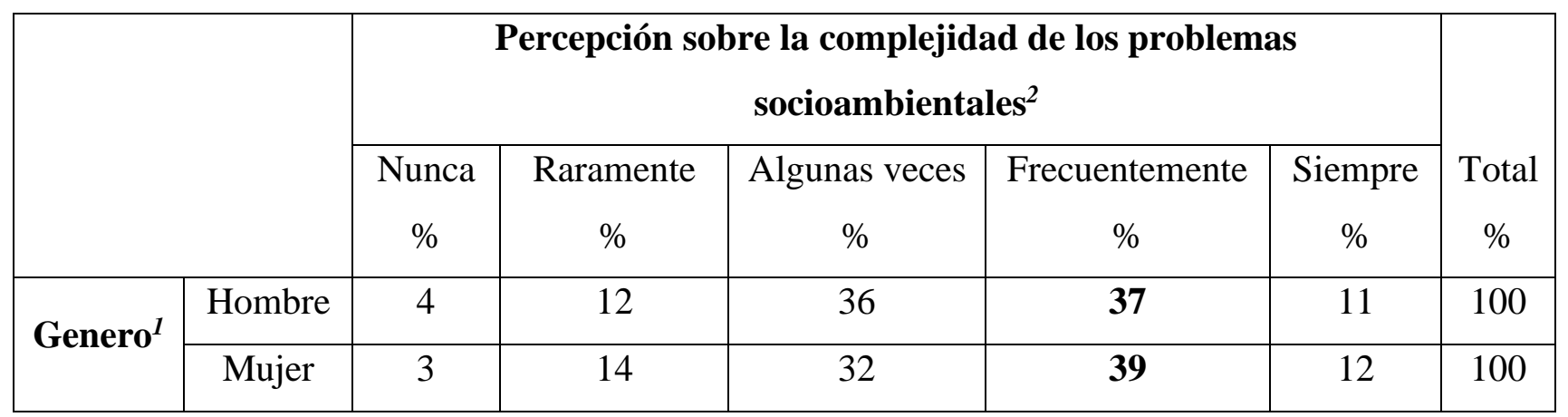

Chi-cuadrado de Pearson $(x 2)=4.979, \mathrm{gl}=4, \mathrm{Sig}=.290, \mathrm{~d}=.026, \mathrm{Sig}=.335$

${ }^{1}$ Variable Independiente $\&^{2}$ Variable dependiente

Fuente: Elaboración propia

Con respecto al análisis de la variable ambientalización en el currículo, los resultados obtenidos mostraron que independientemente de la preparatoria que se trate, un $30 \%$ de los estudiantes encuestados consideraron al currículum con una escasa ambientalización, mientras que un $32 \%$ lo consideró regularmente ambientalizado; un $18 \%$ consideró una buena ambientalización curricular, mientras que $16 \%$ lo consideró con nula ambientalización en los contenidos curriculares; finalmente, un $5 \%$ consideró a los contenidos del currículo excelentemente ambientalizado.

En la figura 2 se pueden observar los porcentajes de los resultados aquí expuestos; además, se ilustra de forma gráfica el comportamiento de las cinco variables trabajadas en el presente estudio. 
Figura 2. Porcentajes de variables de las preparatorias encuestadas de la UAGro

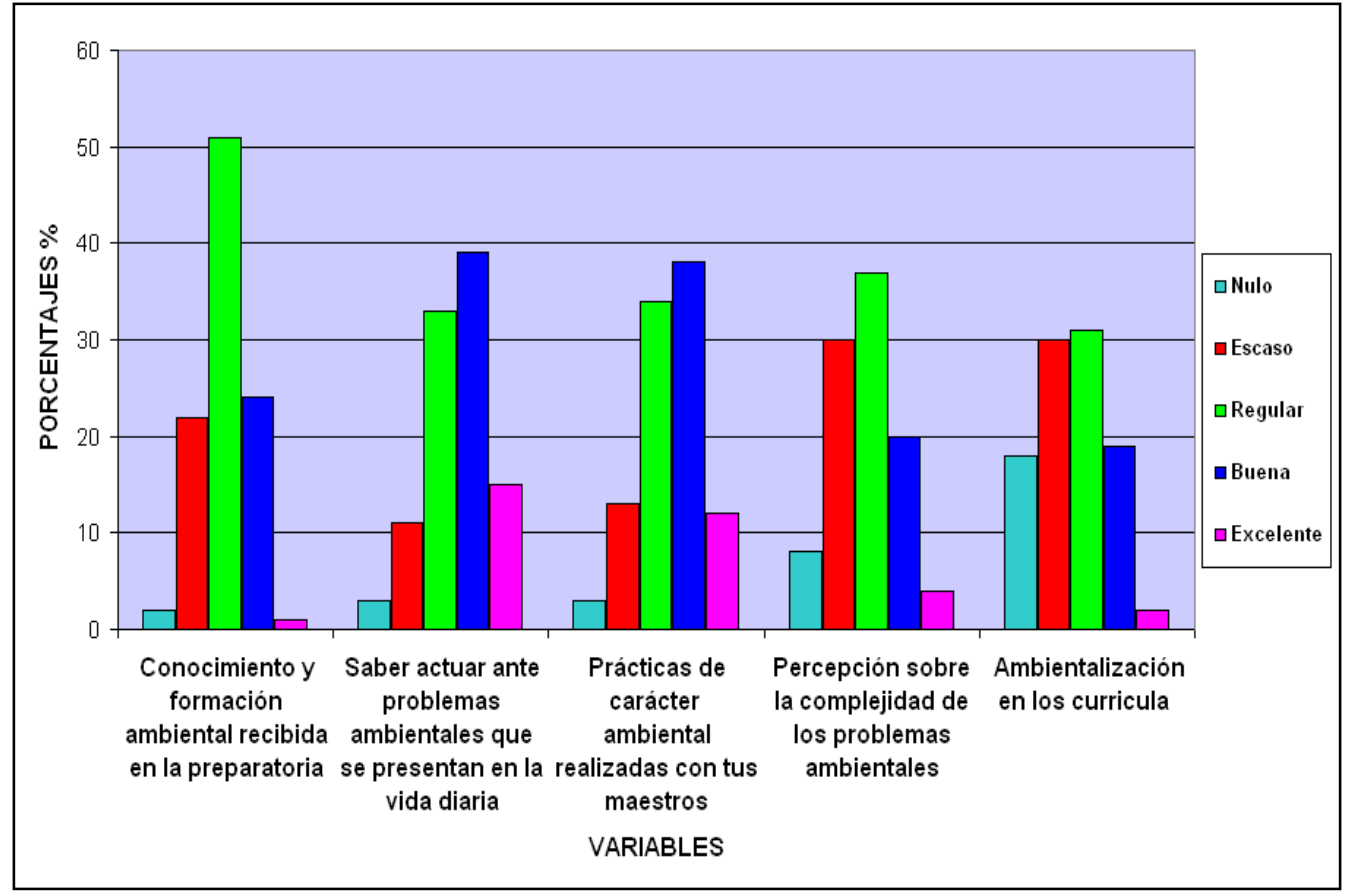

Fuente: Elaboración propia

\section{Discusión}

El análisis de los resultados de la presente investigación demostraron que, independientemente del género, turno y de la preparatoria de que se trate, las frecuencias y porcentajes se determinaron entre las escalas de "escasa", "regular" y "buena", con porcentajes por debajo de la media, lo que ha permitido identificar las áreas de oportunidad para llevar a cabo una intervención metodológica que permita cambiar la percepción que tienen los estudiantes sobre las problemáticas socioambientales actuales y cotidianas. Con respecto al conocimiento y formación ambiental, un $52 \%$ de 1,104 estudiantes encuestados consideraron tener un conocimiento y formación ambiental regular, mientras que un $25 \%$ dijo que fue "buena" y un $22 \%$ "escasa", lo que es justificable debido a la baja ambientalización en el currículo del nivel medio superior; contrastando con el análisis que realizaron Gervacio y Castillo (2019) sobre los contenidos curriculares del NMS de la UAGro, encontraron que solo ecología, biología, geografía y química contienen temáticas relacionadas con el ambiente. En la variable saber actuar ante los problemas socioambientales que se presentan en la vida diaria, un 38\% indicó que saben actuar ante los problemas socioambientales que se les presenta en su vida cotidiana, lo que significa que el $62 \%$ de los estudiantes no están siendo conscientes de las problemáticas ambientales en las que están 


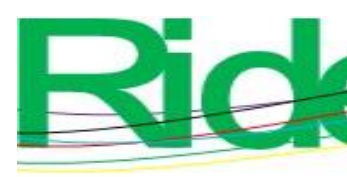

Revista Iberoamericana para la Investigación y el Desarrollo Educativo ISSN $2007-7467$

inmersos en su vida cotidiana, lo que tiene que ver con una baja actividad en sus prácticas de carácter ambiental que realizan con sus profesores.

En la variable percepción sobre la complejidad de los problemas socioambientales, el 38 $\%$ indicó que tienen una percepción "regular", mientras que para el 30 \% es "escasa"; estos resultados permiten inferir sobre cómo perciben los estudiantes las problemáticas ambientales locales y globales.

Aun cuando los resultados indicaron que los estudiantes con frecuencia saben actuar ante los problemas ambientales que se presentan en su vida cotidiana, éstas no se han visto reflejas en acciones ambientales que beneficien a sus escuelas, de acuerdo con lo citado por Castillo y Gervacio (2019) en un estudio de Ecoauditoría aplicado en las escuelas del NMS de la misma Universidad, detectaron la falta de una cultura ambiental y la escasa implementación de estrategias para una correcta gestión ambiental al interior de las escuelas preparatorias bajo estudio.

La educación ambiental es acción, es llevar a la práctica actividades cotidianas a favor de la naturaleza y el bienestar humano, es sinergia con las actividades que se realizan día a día, en la escuela, en casa, o en el lugar de trabajo, debe existir un compromiso, con una apropiada metodología, ejecutarse y verse reflejada en acciones concretas, darle seguimiento y debe considerarse como parte de la vida diaria del individuo; tal como lo afirman Martín-Molero (1999); Wences (2005), quienes concluyeron que el conocimiento no es suficiente: se necesita algo más, como tener conciencia sobre la problemática ambiental y proyectarlo en acciones en beneficio del entorno.

Estudios como los de Coya (2001); Moreno (2005) en el nivel superior, Ignacio y Wences (2006); Calixto (2018) con estudiantes de secundaria, coincidieron con los resultados del presente estudio; donde se demostró que los estudiantes que provienen de las secundarias oficiales cuentan con un importante conocimiento ambiental al llegar a las escuelas preparatorias, esta información no se muestra en los resultados de esta investigación, ya que en la preparatoria tendrían que haber incrementado sus conocimientos ambientales. Otro factor no menos importante que incide en el conocimiento y formación ambiental es la familia, Wences (2005); Ignacio y Wences (2006), concluyeron que, los hogares que reciben más información de carácter ambiental de los medios de comunicación, de las organizaciones de la sociedad civil, de las organizaciones políticas, de familiares y amigos son aquellos cuyos hijos participan más en la defensa del ambiente.

Con respecto a la variable ambientalización en el currículo, los resultados mostraron que son "regulares" a "escasos", al respecto Gervacio y Castillo (2019), en su investigación sobre 


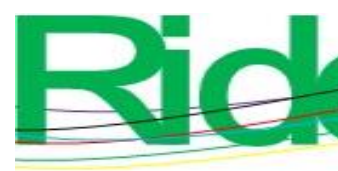

Revista Iberoamericana para la Investigación y el Desarrollo Educativo ISSN $2007-7467$

contenidos relacionados con la dimensión socioambiental en el currículo del nivel medio superior de la UAGro, sus resultados coinciden con la percepción que tienen los estudiantes sobre la ambientalización del currículo universitario; de igual manera, Minor y Ledezma (2011) reconocen que a pesar de los avances por insertar la educación ambiental en el currículo de la educación básica en México, aún falta la incidencia con determinación y voluntad de todos los actores implicados en la toma de decisiones del sistema educativo. De acuerdo con Peza (2014), la educación ambiental para el desarrollo sustentable constituye una herramienta eficaz que "humaniza" la convivencia escolar en la medida que exalta el aprecio por la vida y por el contexto.

Asimismo, Martínez-Fernández y González (2015), a través de un estudio comparativo de los planes ambientales institucionales en 35 universidades en México, encontraron que la comprensión y socialización con estrategias ambientales son laxas, con un bajo impacto en los procesos sustantivos de las universidades, mientras que el estudio de Alfie-Cohen y MartínezFernández (2015) demostró que la estrategia de la sustentabilidad ambiental en sus planes y programas de estudio no ha podido consolidarse debido a que no hay una correcta articulación entre las políticas institucionales y sus estrategias.

Finalmente, se debe enfatizar que la búsqueda de alternativas para solucionar un problema exige la articulación de saberes, lo cual también sucede en el campo ambiental; por ello, puede ser favorable una lectura transversal de conceptualizaciones, métodos y contenidos, ya que el entorno es el escenario cotidiano de construcción de vida, y es allí donde el estudiante interactúa como individuo y como colectivo para reconocerse y reconocer a su mundo, como lo señalan Simões, López y Álvarez (2019). En definitiva, Gervacio y Castillo (2019) recomiendan hacer revisiones permanentes de los programas de estudio para promover la enseñanza-aprendizaje que permita resolver las problemáticas socioambientales actuales y futuras. 


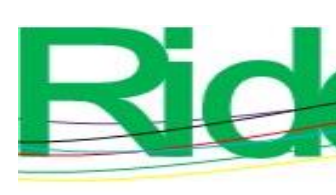

Revista Iberoamericana para la Investigación y el Desarrollo Educativo ISSN $2007-7467$

\section{Conclusiones}

Con base en los resultados aquí expuestos, se considera que los estudiantes del nivel medio superior de la Universidad Autónoma de Guerrero (UAGro) poseen escasos conocimientos y formación ambiental, aun cuando éstos consideren que regularmente saben actuar ante los problemas ambientales, los resultados demostraron una baja actitud proambiental, desconocen las problemáticas socioambientales actuales, se tiene una escasa conciencia para actuar, cuidar y proteger su entorno.

Para lograr cambios significativos en los estudiantes es necesario incidir en su formación moral, en sus valores y principios, en esta tarea los padres deben ser los gestores de estos valores, educando a sus hijos desde casa; la escuela debe hacer lo conducente a través de los planes de estudio, los cuales deben ser rediseñados con base en los nuevos tiempos, las nuevas realidades, las nuevas problemáticas socioambientales que padece actualmente la humanidad, el currículo debe ser actualizado, flexible y contextualizado a la nueva realidad que se está viviendo a nivel global; los docentes en todas la áreas deben ser los facilitadores del conocimiento, de los valores y actitudes en la formación de estudiantes socialmente comprometidos, formados en valores, moral y éticamente responsables con el cuidado de la naturaleza, es fundamental que se instruya a los estudiantes sobre la importancia y la protección del medioambiente en cualquier asignatura que se imparta; las temáticas que se aborden en los planes y programas deben llevar un enfoque integrador multidisciplinario y transversalizado, que los aprendizajes sean significativos, que ayuden a resolver problemas reales, de la vida cotidiana, de tal manera que los estudiantes lleven a la práctica la teoría aprendida en clase y se convierta en aprendizajes útiles para la vida; en este sentido, los docentes tienen una función relevante, una responsabilidad profesional y moral, una tarea importante en el diseño de estrategias didáctico-pedagógicas que permita involucrar la mayor parte de las asignaturas y trabajar colaborativamente entre los docentes con distintos perfiles para tener un programa exitoso. Los contenidos deben ser con un enfoque socioambiental; es decir, la información ambiental debe ofrecerse con suficientes garantías de veracidad y rigor científico, pasando de la observación a la acción resolviendo problemas existentes en la propia escuela y en su entorno más cercano. A las escuelas se les debe proporcionar las herramientas metodológicas y logísticas para que se sumen a movimientos ambientales, es necesario la participación de toda la comunidad educativa, los padres de familia, comunidades aledañas y las entidades gubernamentales para que sea un trabajo en conjunto y los resultados sean los deseados. 

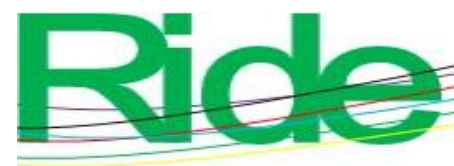

Revista Iberoamericana para la Investigación y el Desarrollo Educativo

ISSN $2007-7467$

Por otro lado, la propuesta de diseño y desarrollo curricular deberá considerar los elementos prevalecientes en el contexto internacional, nacional y regional con la finalidad de ajustarlos al contexto en el cual se encuentran inmersos los estudiantes. En definitiva, es fundamental que los estudiantes aprendan a pensar críticamente, reflexivamente en situaciones de crisis y proponer alternativas viables para mejorar y transformar su realidad. Por eso, adquieren significado todos los elementos de innovación curricular que apuntan al establecimiento de vínculos entre la escuela y la sociedad, como binomio que deberá proyectar sus esfuerzos para contribuir a insertar al alumnado en el contexto socioambiental, a partir del análisis y comprensión de las complejas relaciones que se establecen y ante las cuales se espera que puedan actuar de manera creativa y propositiva como sujetos autónomos. Lo ideal sería generar un currículo integral y holístico centrado, desde luego, en el perfil de egreso del estudiante de bachillerato.

Finalmente, cabría una sólida revisión para integrar a los programas de estudio ejes ambientales claros y bien definidos, que sirvan como base para trabajar la educación ambiental en las escuelas, si bien está considerado "el cuidado al medio ambiente" como nuevo perfil de egreso del estudiante de bachillerato, no se especifica la estrategia para lograr esta competencia, ya que la indicación es "piensa global y actúa local", una máxima que no ayuda de nada si no se tiene una estrategia metodológica bien definida. 


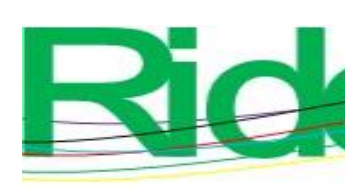

Revista Iberoamericana para la Investigación y el Desarrollo Educativo ISSN 2007-7467

\section{Referencias}

Alfie-Cohen, M. y Martínez-Fernández, C. N. (2015). La UAM Cuajimalpan: reflexiones en su décimo aniversario. Revista de la Educación Superior, 44(176), 37-61 Recuperado de https://www.elsevier.es/es-revista-revista-educacion-superior-216-pdf-

S0185276015001181

Benayas del Álamo, J., Alba, D. y Sánchez, S. (2002). La ambientalización de los campus universitarios. El caso de la Universidad Autónoma de Madrid. Ecosistemas, 11(3). Recuperado de https://www.revistaecosistemas.net/index.php/ecosistemas/article/view/601

Calixto, F. R. (2018). Representaciones en torno al cambio climático de los estudiantes de una escuela secundaria. Ciudad de México: Universidad Pedagógica Nacional.

Calixto, F. R. (2019). Estrategias didácticas sobre el medio ambiente y el cambio climático. CD de México: Universidad Pedagógica Nacional.

Carrasco, L. M. E. y Vásquez, R. E. (2016). La educación ambiental: un saber necesario en la formación universitaria. Primer Congreso Nacional de Educación Ambiental para la Sustentabilidad. Eje: EAS en las instituciones educativas. Academia Nacional de Educación Ambiental ANEA. Recuperado de http://www.anea.org.mx/CongresoEAS/Docs/332P-INST-CarrascoLozanoV2(corr).pdf

Castillo, E. B. y Gervacio, J. H. (2019). Ecoauditoria ambiental aplicada a centros escolares del nivel medio superior de la Universidad Autónoma de Guerrero. REMEA- Revista Eletrônica do Mestrado em Educação Ambiental, 36(2), 319-341. Doi: https://doi.org/10.14295/remea.v36i2.9139

Coya, G. M. (2001). La ambientalización de la universidad: un estudio sobre la formación ambiental de los estudiantes de la Universidad de Santiago de Compostela y la política ambiental (tesis doctoral). Facultad de Ciencias de la Educación, Departamento de Teoría e Historia de la Educación de la Universidad de Santiago de Compostela.

Gervacio, J. H. y Castillo, E. B. (2019). Dimensión socioambiental en los contenidos del currículo del nivel medio superior de la Universidad Autónoma de Guerrero. RIDE-Revista Iberoamericana para la Investigación y el Desarrollo Educativo, 10(19), 1-25. Doi: https://doi.org/10.23913/ride.v10i19.500 


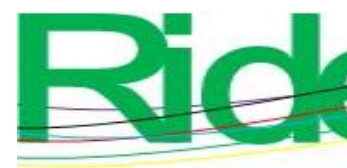

Revista Iberoamericana para la Investigación y el Desarrollo Educativo ISSN 2007-7467

González-Gaudiano, É. J. y Arias-Ortega, M. Á. (2017). La formación de educadores ambientales en México: avances y perspectivas. Educar em Revista, (63), 53-66. Doi: https://doi.org/10.1590/0104-4060.49136

Hernández-Sampieri, R., Fernández, C. C. y Baptista, L. P. (2014). Metodología de la investigación (6. ${ }^{\mathrm{a}}$ ed.). México: Editorial Mc. Graw Hill.

Ignacio, G. B. y Wences R. (2006). Aprendizaje de la problemática ambiental en las escuelas secundarias. En Gasca Zamora, J. (coord.), La construcción de perspectivas de desarrollo en México desde sus regiones. Mérida Yucatán, (México): Asociación Mexicana de Ciencias para el Desarrollo Regional A.C. (AMECIDER).

Leff, E. (2006). La universidad y la formación ambiental. Diez líneas de acción. Revista Educación $\begin{array}{lllll}\text { Superior } & y & \text { Sociedad, } & \text { 21-25. } & \text { Recuperado }\end{array}$ de http://ess.iesalc.unesco.org.ve/index.php/ess/article/viewFile/165/129

Martínez-Fernández, C. N. y González, G. E. J. (2015). Las políticas para la sustentabilidad de las instituciones de educación superior en México: entre el debate y la acción. Revista de la Educación Superior, 44(174), 61-47. Recuperado de http://publicaciones.anuies.mx/pdfs/revista/Revista174_S3A3ES.pdf

Martín-Molero, F. (1999). Educación ambiental. Madrid: Editorial Síntesis S.A.

Medina, A. y Páramo, P. (2014). La investigación en educación ambiental en América Latina: un análisis bibliométrico. Revista Colombiana de Educación, 66, 19-72. Recuperado de http://www.scielo.org.co/pdf/rcde/n66/n66a03.pdf

Minor, C. y Ledezma, A. (2011). La educación básica a la luz de Tbilisi +31 en Visiones Iberoamericanas de la educación ambiental en México. En Súcar, S. (coord.), Memorias del Foro Tbilisi + 31. Universidad de Guanajuato.

Moreno, L. E. (2005). La formación inicial en educación ambiental de los profesores de secundaria en periodo formativo (tesis doctoral). Escuela Universitaria del Departamento de Didáctica de las Ciencias Experimentales y Sociales de la Universidad de Valencia.

Organización de las Naciones Unidas para la Educación, la Ciencia y la Cultura [Unesco] (2014). Declaratoria de Aichi-Nagoya sobre la educación para el desarrollo sostenible. Organización de las Naciones Unidas para la Educación, la Ciencia y la Cultura (United Nations Educational, Scientific and Cultural Organization). Recuperado de https://unesdoc.unesco.org/ark:/48223/pf0000231074_spa 


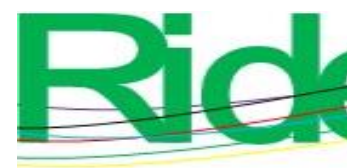

Revista Iberoamericana para la Investigación y el Desarrollo Educativo ISSN $2007-7467$

Organización de las Naciones Unidas para la Educación, la Ciencia y la Cultura [Unesco] (2015). Educación para la ciudadanía mundial: temas y objetivos de aprendizaje. Organización de las Naciones Unidas para la Educación, la Ciencia y la Cultura (United Nations Educational, Scientific and Cultural Organization). Recuperado de https://unesdoc.unesco.org/ark:/48223/pf0000233876

Organización Universitaria Interamericana - Organización Interamericana para la Educación Superior [OUI-IOHE] (2011). Declaración de las Américas: por la sustentabilidad de y desde la universidad. Recuperado de http://www.uv.mx/cosustenta/files/2012/09/declaracion.pdf

Pérez, L. C. (2009). Técnicas estadísticas con SPSS (Statistical Package for the Social Sciences V16). Prentice Hall Pearson.

Peza, H. G. (2014). Educación para el desarrollo sustentable: problemas ambientales, estrategias pedagógicas y recursos didácticos. México: Instituto de Investigación, Innovación y Estudios de Posgrado para la Educación (IIEPE), Secretaría de Educación del Gobierno del Estado de Nuevo León y Secretaría de Medio Ambiente y Recursos Naturales.

Secretaría de Educación Pública [SEP] (2017). Los fines de la educación en el siglo XXI. Recuperado de https://www.planyprogramasdestudio.sep.gob.mx/descargables/biblioteca/basica-educfisica/II-LOS-FINES-DE-LA-EB.pdf

Simões, A. S, Yanes López, G., y Álvarez Díaz, M. (2019). Transversalidad de la educación ambiental para el desarrollo sostenible. Universidad y Sociedad, 11(5), 25-32. Recuperado de http://rus.ucf.edu.cu/index.php/rus

Tovar-Gálvez, J. C. (2012). Fundamentos para la formación de líderes ambientales comunitarios: consideraciones sociológicas, deontológicas, epistemológicas, pedagógicas y didácticas. Revista Luna Azul, (34), 214-239. Recuperado de https://www.redalyc.org/articulo.oa?id=3217/321727348013

Weigel, R. H. and Weigel, J. (1978). Environmental concern. The development of measure. Environment and Behavior 10(1):3-15

Wences, R. R. (2005). Opinión, conciencia y acción ambientalistas de los acapulqueños. En Memorias del X Congreso Nacional y IV Congreso Internacional de Ciencias Ambientales. Academia Nacional de Ciencias Ambientales. Chetumal, Quintana Roo, México. 


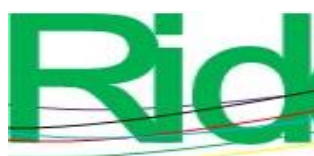

Revista Iberoamericana para la Investigación y el Desarrollo Educativo ISSN 2007 - 7467

Yazan, B. (2015). Three Approaches to Case Study Methods in Education: Yin, Merriam, and Stake. The Qualitative Report, 20(2), 134-152. Recuperado de https://nsuworks.nova.edu/tqr/vol20/iss2/12

Zúñiga-Sánchez, O. y Marúm-Espinosa, E. (2016). La educación ambiental para la sustentabilidad en las instituciones de educación superior. Una aproximación conceptual. En Memorias del ler Congreso Nacional de Educación Ambiental para la Sustentabilidad. Asociación Nacional para la Educación Ambiental (ANEA). Recuperado de http://www.anea.org.mx/CongresoEAS/Docs/337P-INST-EspinosaV2(corr).pdf 


\begin{tabular}{|c|c|}
\hline Rol de Contribución & Autor (es) \\
\hline Conceptualización & Herlinda Gervacio Jiménez \\
\hline Metodología & $\begin{array}{l}\text { Herlinda Gervacio Jiménez (igual), Benjamín Castillo Elías } \\
\text { (igual) }\end{array}$ \\
\hline Software & Herlinda Gervacio Jiménez \\
\hline Validación & $\begin{array}{l}\text { Herlinda Gervacio Jiménez (igual), Benjamín Castillo Elías } \\
\text { (igual) }\end{array}$ \\
\hline Análisis Formal & $\begin{array}{l}\text { Herlinda Gervacio Jiménez (igual), Benjamín Castillo Elías } \\
\text { (igual) }\end{array}$ \\
\hline Investigación & $\begin{array}{l}\text { Herlinda Gervacio Jiménez (igual), Benjamín Castillo Elías } \\
\text { (igual) }\end{array}$ \\
\hline Recursos & $\begin{array}{l}\text { Herlinda Gervacio Jiménez (igual), Benjamín Castillo Elías } \\
\text { (igual) }\end{array}$ \\
\hline Curación de datos & Herlinda Gervacio Jiménez \\
\hline $\begin{array}{l}\text { Escritura - Preparación del } \\
\text { borrador original }\end{array}$ & $\begin{array}{l}\text { Herlinda Gervacio Jiménez (igual), Benjamín Castillo Elías } \\
\text { (igual) }\end{array}$ \\
\hline $\begin{array}{l}\text { Escritura - Revisión } \quad y \\
\text { edición }\end{array}$ & $\begin{array}{l}\text { Herlinda Gervacio Jiménez (igual), Benjamín Castillo Elías } \\
\text { (igual) }\end{array}$ \\
\hline Visualización & $\begin{array}{l}\text { Herlinda Gervacio Jiménez (igual), Benjamín Castillo Elías } \\
\text { (igual) }\end{array}$ \\
\hline Supervisión & $\begin{array}{l}\text { Herlinda Gervacio Jiménez (igual), Benjamín Castillo Elías } \\
\text { (igual) }\end{array}$ \\
\hline $\begin{array}{l}\text { Administración } \\
\text { Proyectos }\end{array}$ & $\begin{array}{l}\text { Herlinda Gervacio Jiménez (igual), Benjamín Castillo Elías } \\
\text { (igual) }\end{array}$ \\
\hline Adquisición de fondos & $\begin{array}{l}\text { Herlinda Gervacio Jiménez (igual), Benjamín Castillo Elías } \\
\text { (igual) }\end{array}$ \\
\hline
\end{tabular}

